\title{
Hybrid system design for platoon collaborative strategies
}

\author{
HUANG Zichao ${ }^{1, a}$, WU Qing ${ }^{1, b}$, MA Jie ${ }^{2, c}$ \\ ${ }^{1}$ School of Logistics Engineering, Wuhan University of Technology; Wuhan, Hubei, china; \\ ${ }^{2}$ School of Navigation, Wuhan University of Technology, Wuhan, Hubei 430063, China \\ ahzc@whut.edu.cn, ’bwq@whut.edu.cn, ํmajie@whut.edu.cn
}

Keywords: hybrid system, hybrid automaton, MPC, Stateflow

Abstract. Collaborative control of the autonomous platoon poses significant theoretical and technical challenges. The processes of the platoon collaborative control need to deal with the discrete strategies switching and handle the continuous dynamic behavior of the vehicles. While, the platoon control system exhibits both continuous and discrete dynamic behavior, it can be modeled by a hybrid automaton of collaborative strategies. In this paper, a collaborative strategies hybrid automaton is applied to implement platoon cooperative control. Firstly, A MPC controller is set up to generate the trajectories of the control parameters. Afterwards, constructing the collaborative strategies hybrid automaton to implement the transition between different collaborative strategies (such as cruise, follow, merge and split). The prominent advantage of the proposed approach is that it can deal with the continuous and the discrete dynamic simultaneously to keep the whole system functioning well in different traffic condition. To validate the proposed approach, some simulations are conducted using the Stateflow tool box in SIMULINK, and the performance of the proposed method is verified.

\section{Introduction}

Highway congestion is an increasing problem, especially in and around urban areas. The platooning concept assumes that traffic on the highway is organized in groups of tightly spaced vehicles. There are various advantages for organizing the vehicles as platoon, such as increasing road capacity and efficiency, reducing congestion energy consumption and pollution, and enhancing safety and comfort [1]. Therefore, collaborative driving for platoon has become a hot topic in the field of Intelligent Vehicle-Highway System (IVHS). Yi et al. proposed a platoon collaborative driving system structure, employed the spring dampers to enhance system stability to implement the platoon collaborative driving system [2]. Considering platoon uncertainty and disturbance, Hwang et al. used the double time discrete control based on singular perturbation theory and divided the platoon into slow and fast subsystems. He then controlled V2V distance tolerance change and completed platoon following strategy [3]. At present, the construction and realization of platoon collaborative driving system mainly focus on structure of IVHS (Intelligent Vehicle Highway System) research and hybrid dynamic system control. In IVHS structure, PATH research group of University of California, Berkeley, has proposed five layers structure of IVHS [4]. The top two layers, called network layer and link layer, belong to the roadside and are primarily concerned with the macro control of the traffic flow, while the bottom two, called coordination layer and regulation layer, reside on the vehicles and are primarily concerned with safety. The physical layer contains the plant (i.e. the vehicles and highway, with their sensors, actuators and communication equipment) which is not part of the controller. While, the interaction between the coordination layer (which is primarily discrete) and the regulation layer (which is 
primarily) gives rise to hybrid dynamics $[5,6]$.

In this paper, we propose a hybrid structure to describe the platoon collaboration system and enable effective switching between the different collaborative strategies. The main advantage of this method is that the collaborative strategies can now be performed more efficiently and safely since all possible next feasible state from a current state can be searched on a discrete graph and the control trajectory is guarded by both the discrete command and the continuous control. The continuous part of the system can be regarded as a trajectories generation problem. A MPC approach is applied to deal with the multi-objective and multi-constrain optimization to generate the strategy transition control trajectories which can solve the problem more efficiently especially for a large group of vehicles $[7,8,9]$.

This paper is organized as follows, In Section 2 we put forward a MPC controller to generation the vehicle control trajectory for collaborative strategies. The hybrid automaton is detailed in Section 3. In section 4, we give the simulation result to validate the performance of the proposed methods. Conclusions are given in Section 5.

\section{Vehicle Control Trajectory Generation for Platoon Collaborative strategies}

Implementing the vehicle control trajectory generation is to deal with the continuous part of the hybrid system. In order to study this problem, we first if the problem is feasible assuming that all of the information about the vehicle is accessible, including the vehicle's own state and the surrounding environment information.

Consider the vehicles in the platoon system each with the following kinematic model,

In the rear axle pivot point $(\mathrm{X}, \mathrm{Y})$, the speed $\mathrm{v}$ is presented as,

$$
v=\mathcal{K}_{\cos \varphi+\mathscr{s i n} \varphi}
$$

$\chi=[x, y, \varphi]^{T}$ denote the state vector of the host vehicle, $\mathrm{x}, \mathrm{y}$ denote the Cartesian coordinates of the vehicle's position, $\varphi$ denotes the counterclockwise orientation of the vehicle from the x-axis. $u=[v, \delta]^{T}$ denote the vehicle's input vector, where $\mathrm{v}$ denotes the speed, $\delta$ denotes the front wheel steering angle. The vehicle kinematic model $\chi \alpha=f(\chi, u)$ is given as

$$
\chi \alpha=\left[\begin{array}{c}
\alpha \\
\phi \\
\phi
\end{array}\right]=\left[\begin{array}{c}
\cos \varphi \\
\sin \varphi \\
\tan \delta_{f} / l
\end{array}\right] v
$$

Define the vehicle's configuration at time $t$ as $g(t)=\left[x^{T}(t), u^{T}(t)\right]^{T}$ which specifies the state and input conditions for the vehicle in the platoon at time $t$. The admissible input for each vehicle is specified by an input constraint as, $\|\mathscr{L}(t)\| \leq \alpha, \forall t \in[0, T],\|\delta \&(t)\| \leq \omega, \forall t \in[0, T]$, Where $\alpha$ is the maximum allowable acceleration input at any time and $\delta$ denote the front wheel steering angle.

We notice that the trajectory generation is a multi-objective and multi-constraint problem. In this paper we applied MPC method to realize the collaborative strategies which, in fact, be formulated as an optimization problem. The objective is to determine the parameters for the input trajectories, minimizing the cost function, subject to dynamical, configuration constraints.

The optimization problem used to derive the trajectory parameters that minimize the cost $J_{i}$, different $J_{i}$ leads to different control effect which corresponding different collaborative strategies. For example, we defined a $J_{\text {Cruise }}$ for the cruise strategies shown as blow, 


$$
J_{\text {Cruise }}=\frac{S_{o b s} v_{i}}{\left(x_{i}-x_{0}\right)^{2}+\left(y_{i}-y_{0}\right)^{2}+\zeta}
$$

Where $S_{\text {obs }}$ stands for the weight coefficient; $\zeta$ is a lesser integer which can prevent the denominator from being zero; $\left(\mathrm{x}_{\mathrm{i}}, \mathrm{y}_{\mathrm{i}}\right)$ stands for the position coordinate in the vehicle coordinate system; $\left(\mathrm{x}_{0}, \mathrm{y}_{0}\right)$ stands for vehicle centroid coordinate. The control objectives in the trajectory planning layer not only reduce the deviation from the overall referential path as much as possible, but also avoid obstacles. Therefore, the optimization object is shown as blow:

$$
\min _{U_{t}} \sum_{i=1}^{N_{P}}\left\|\eta(t+i \mid t)-\eta_{\text {ref }}(t+i \mid t)\right\|_{Q}^{2}+\left\|U_{i}\right\|_{R}^{2}+J_{o b s, i}
$$

Where $\mathrm{J}_{\mathrm{obs}, \mathrm{i}}$ stands for the obstacle avoidance function on sampling time. The nonlinear objective function can facilitate the programing of the obstacle avoidance function. So there is no point in linearization processing. The nonlinear dynamics model is adopted to directly solve the model instead.

\section{Hybrid Structure for Platoon coordination strategies}

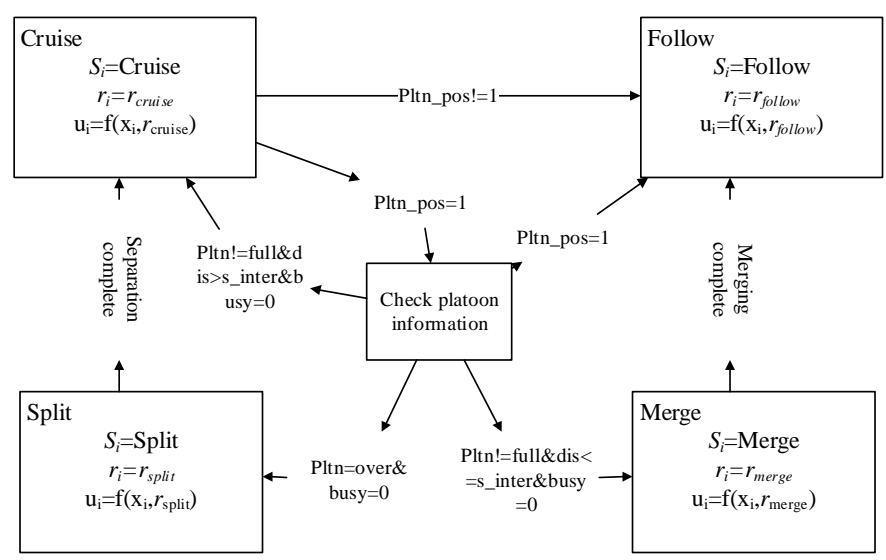

Fig. 1 Platoon collaborative hybrid automaton

The collaborative relationship between host vehicle and the surrounding vehicles can be divided into either two inter- or intra-platoon. While the platoon collaboration process can be regarded as a combination of switching from different discrete collaborative strategies and deal with the continuous dynamic correspondingly. We will use a hybrid automaton to describe this system. The hybrid automaton can handle the strategies determining and the car control synchronously which defined as a collection, $\mathrm{D}=\left(Q_{D}, X_{D}, V_{D}, Y_{D}\right.$, Init $_{D}, f_{D}, h_{D}$, inv $\left.v_{D}, E_{D}, G_{D}, R_{D}\right)$. The discrete state of the strategies determining is defined by: $Q_{D}=\left\{q_{D}\right\}, Q_{D}=\{$ CRUISE, FOLLOW, MERGE, SPLIT $\}$, and $X_{i}=\left\{x_{i}, y_{i}, \varphi_{i}\right\}, \mathrm{X}_{i}={ }^{3}$ which denote the continuous state of the $\operatorname{car}_{\mathrm{i}} . V_{i}=\left\{v_{i}, \delta_{i}, d_{i}, S_{i}\right\}$, $\mathrm{V}_{i}={ }^{3} \times Q_{D}$, is a finite collection of input variables $Y_{i}=\left\{\chi_{i}, S_{i}\right\}, \mathrm{Y}_{i}={ }^{3} \times Q_{D}$ is a finite collection of output variables. And both the $V_{i}$ and $Y_{i}$ contain the discret and the continuous variables. The initial states is set as, Init $=\{C R U I S E\} \times\left[x_{0}, y_{0}, \varphi_{0}\right], f\left(q_{i}, x_{i}, y_{i}, \varphi_{i}, v_{i}, \delta_{i}\right)=A x+B u$

is a vector field. The $i n v_{D}$ assigns to each $q_{D}$ an invariant set.; $E \subset Q \times Q$ is a collection of discrete transitions, $\mathrm{G}$ assigns to each $e \in E$ and $x \in X$ a guard; and $\mathrm{R}$ assigns to each $e \in E$ and $x \in X$ a reset relation. In Figure 2, the collaborative hybrid automaton is shown in detail. 
It must be notice that the proposed automaton requires a strong synchronous assumption made on communication capabilities of the vehicles for exchanging events.

\section{Experimental Results}

In order to test the proposed method, we perform a series of simulation on the platform which construct by stateflow tool box in SIMULINK.

In the first simulation, we set about cruise strategy simulation for a single car. We assigned a desired trajectory, meanwhile, some obstacles are set on it. The simulation results are shown in Figure 4. We can see the host car track the desired trajectory and avoid the obstacle.

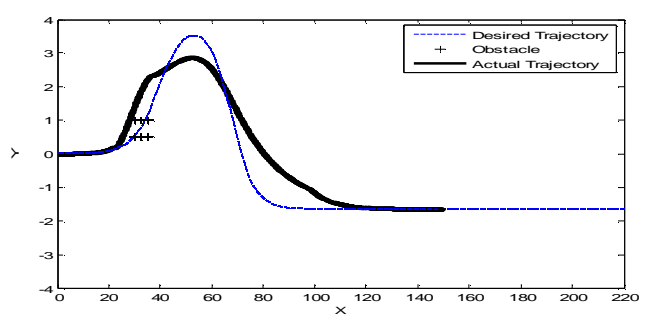

Fig. 2 Experimental results of Vehicle Cruise tracks

In the second simulation, we set a scenario which containing three car, namely, Car1, Car2 and Car3 in a straight road. Set the initial position of Car1, Car2 and Car3 in 0m, 35m and 85m respectively. Set the initial speed of Car1 as $10 \mathrm{~m} / \mathrm{s}$ and desired speed of Car1 is $11.1 \mathrm{~m} / \mathrm{s}$. Set the initial speed of Car2 as $5 \mathrm{~m} / \mathrm{s}$ and desired speed of Car 1 is $16.7 \mathrm{~m} / \mathrm{s}$. Set the initial speed of Car3 as $0 \mathrm{~m} / \mathrm{s}$ and desired speed of Car1 is $16.7 \mathrm{~m} / \mathrm{s}$. Let all of the three cars are in a same platoon and the distance of intra-platoon is $20 \mathrm{~m}$
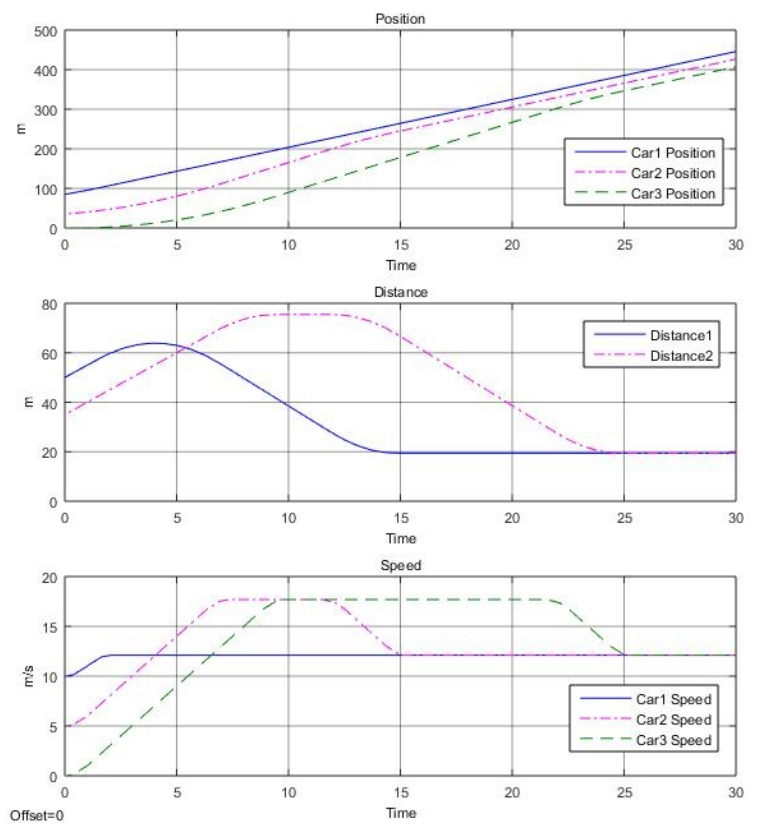

Fig. 3 Experimental results of Cruise strategies change to Follow strategies

We can see in the first stage, all of the three cars did not achieve the desired speed, they execute the cruise strategy, so they began to accelerate under constrains of the acceleration. Car1 reached the desired speed first and keep the speed, meanwhile, Car2 and Car3 went on accelerate until to achieve their desire speed. Due to the initial position and the desired speed, the Car2 caught up with Car1 first and began to decelerate until the Car1's speed. And then, Car3 approaching the front car and implement the same control to keep the setting distance. At last three car merged into a platoon 
and stay in the follow strategy.

\section{Conclusion}

In this paper, we analysis the hybrid characteristic of the platoon collaborative driving system and construct a platoon collaborative strategies hybrid automaton. As a result, a MPC control approach is implement to deal with the multi-objective and multi-constrain optimization problem, which achieving the control trajectory generation for a given initial state and the target state. The proposed a solution to implement the platoon collaborative strategies using a hybrid automaton model. The main contribution can be summarized as follows: 1) A MPC approach is set up to deal with the continuous dynamic behavior of the vehicle, which can represent the problem as a multi-objective and multi-constraint optimization and be solved more efficiently. 2) The proposed hybrid automaton can implement the transition between different collaborative strategies (such as cruise, follow, merge and split) and keep the whole system functioning well in different traffic condition. At last, construct the simulation platform in SIMULINK with stateflow tool box to validate the proposed approach. Some simulations are conducted and the results show that the proposed approach can work well under complex driving conditions.

\section{Acknowledgements}

This work was financially supported by the National Natural Science Foundation of China (No.61174173, No.61203236)

\section{References}

[1] Michaud F, Lepage P, Frenette P, et al. Coordinated maneuvering of automated vehicles in platoons [J]. Intelligent Transportation Systems, IEEE Transactions on, 2006, 7(4): 437-447.

[2] Yi Soo-Yeong, Chong Kil-To. Impedance control for a vehicle platoon system[J]. Mechatronics, 15(5), 2005:627-638.

[3] Hwang Kab-Ju, Yurkevich, V.D..Vehicle Following Longitudinal DecentralizedFeedback Control Based on Two-Time-Scale Motions[C]. The 1st International Forum on Strategic Technology,Ulsan,Korea, Oct.18-20, 2006:5a-9.

[4] Horowitz, R.,Varaiya, P..Control design of an automated highway system[J]. Proceedings of the IEEE, 88(7), 2000: 913-925

[5] Girault Alain. A hybrid controller for autonomous vehicles drivingon automated highways[J]. Transportation Research Part C: Emerging Technologies, 12(6), 2004: 421-452.

[6] Morari, M..Hybrid Systems: Theory, Computation andApplications[C].The 6th World Congress on Intelligent Controland Automation, Dalian, China, Jun.21-23, 2006: I

[7] Choi C, Kang Y, Lee S. Emergency collision avoidance maneuver based on nonlinear model predictive control. In: Proceedings of IEEE International Conference on Vehicular Electronics and Safety (ICVES), 2012.IEEE; 2012. p. 393-8.

[8] Chen Y, Zhang D, Li K. Enhanced eco-driving system based on v2x communication. In: Proceedings of the 15th International IEEE Conference on Intelligent Transportation Systems (ITSC), 2012. IEEE; 2012.p. 200-5.

[9] ] Cao W, Mukai M, Kawabe T, Nishira H, Fujiki N. Cooperative vehicle path generation during merging using model predictive control with real-time optimization. Control Eng. Prac. 2015;34:98-105. 